\title{
Maximum Likelihood Segmentation with Rayleigh Distribution of Ultrasound Images
}

\author{
A Sarti, C Corsi, E Mazzini, C Lamberti \\ Department of Electronics, Computer Science and Systems, University of Bologna, Italy
}

\begin{abstract}
This study presents a geometric model for segmentation of ultrasound images. A partial differential equation based flow is designed in order to achieve a maximum likelihood segmentation of the target in the scene. The flow is derived as the steepest descent of an energy functional taking into account the density probability distribution of the gray levels of the image as well as smoothness constraints. To model gray level behavior of ultrasound images the classic Rayleigh probability distribution is considered. The steady state of the flow presents a maximum likelihood segmentation of the target. A finite difference approximation of the flow is derived and numerical experiments are provided. Results are presented on ultrasound medical images.
\end{abstract}

\section{Introduction}

Ultrasound images are difficult candidates for shape recovery because of low signal/noise ratio which greatly reduces the observable details within such images. Recently several methods based on evolution of partial differential equations have been proposed to segment relevant structures of interest. The classical active contour models (snakes) are based on the evolution of a curve attracted by image boundaries in order to detect objects [1]. Malladi and Sethian implemented active contour models with robust numerical techniques based on level set methods. Level set methods introduced first by Osher and Sethian [2], have been extensively used to track the evolution of fronts in a variety of applications. These methods embed the desired interface as the zero level set of an implicit function and then employ finite differences to approximate the solution of the partial differential equation. The aforementioned approaches for segmentation are "edge-based", i.e. they evolve a curve with a speed function depending on a pre-computed edge indicator. It's very effective in case edges are true discontinuities in the image and the signal to noise ratio is not too low. Recently a "region-based" level set based method has been proposed by Chan and Vese to segment images with smooth or vanishing boundaries [3]. In this approach the speed of curve evolution involves integral quantities (like mean values of the gray level image pixels inside and outside the curve) instead of differential quantities (like image gradient). This approach is very effective in case of smooth edges or low signal/noise ratio.

In this study we take a different view of the segmentation problem by keeping the region based approach of Chan-Vese and embedding in the segmentation model the a priori knowledge of statistical distribution of grey levels in medical images [4]. In particular concerning ultrasound images, we note that image pixels are modelled as Rayleigh distributed random variables. Then the proposed method drives the curve evolution to achieve a maximum likelihood segmentation of the target, with respect to the statistical distribution law of image pixels.

\section{Methods}

Let us consider an image $I$ as a real positive function defined in a rectangular domain $\Omega \subset \Re^{2}$. The gray levels are assumed to be uncorrelated and independently distributed. They are thus characterized by their respective probability density. Now we define a closed curve $C$ partitioning the image domain in an "inside" $\Omega_{\mathrm{i}}$ and an "outside" $\Omega_{\mathrm{e}}$ and denote with $\mathrm{P}_{\mathrm{i}}=\prod_{\Omega \mathrm{i}(C)} \mathrm{p}(I)$ the probability of the random field inside the curve and with $\mathrm{P}_{\mathrm{e}}=\prod_{\Omega \mathrm{e}(\mathrm{C})} \mathrm{p}(I)$ the probability outside the curve. Without any a priori knowledge about the shape of the object to be detected, we look for the curve $C$ that maximizes the likelihood function given by the product of the inner and the outer probability [5]: $\mathrm{P}[I \mid C]=\mathrm{P}_{\mathrm{i}} \mathrm{P}_{\mathrm{e}}$. Since the $\log$ function is strictly increasing, the maximum value of $\mathrm{P}[I \mid C]$, if it exists, will occur at the same points as the maximum value of $1(I, C)=\log (\mathrm{P}[I \mid C])$. This function is the "log likelihood" and in many cases it is easier to work with it than with the likelihood function. Indeed, the product structure of the probability function is transformed in a summation or integral structure of the log likelihood. Passing to the continuous limit, we replace the sum with the integral and, to perform a maximum likelihood segmentation of the target, we need to maximize the functional $l$ with respect to variation of the 
curve $C$. By considering the Rayleigh noise distribution: $\mathrm{p}(I)_{\text {Rayleigh }}=I(\mathrm{x}, \mathrm{y}) / \sigma^{2} \exp \left(-\mathrm{I}(\mathrm{x}, \mathrm{y})^{2} / \sigma^{2}\right), \quad$ the corresponding log-likelihood is given by [6]:

$$
\begin{aligned}
& l(I, C)=\log P_{i}+\log P_{e}= \\
& =\int_{\Omega_{i}(C)} \log p(I) d x d y+\int_{\Omega_{e}(C)} \log p(I) d x d y= \\
& =A_{i} \log \left(\frac{1}{A_{i}} \int_{\Omega_{i}(C)} I(x, y)^{2} d x d y\right)+A_{e} \log \left(\frac{1}{A_{e}} \int_{\Omega_{e}(C)} I(x, y)^{2} d x d y\right)
\end{aligned}
$$

where $\mathrm{A}_{\mathrm{i}}$ e $\mathrm{A}_{\mathrm{e}}$ are respectively the number of pixels in $\Omega_{\mathrm{i}}$ and $\Omega_{\mathrm{e}}$ the parameters of the probability density function (pdf) have been estimated following Chesnaud [6].

In order to obtain a well-posed and well-conditioned problem we need to introduce a regularization in the shape of the curve. To this scope, a length term has been introduced as in [3], and finally we ask to minimize the functional:

$$
\begin{aligned}
& F=\mu(\text { length }(C))-A_{i} \log \left(\frac{1}{A_{i}} \int_{\Omega_{i}(C)} I(x, y)^{2} d x d y\right)+ \\
& +A_{e} \log \left(\frac{1}{A_{e}} \int_{\Omega_{e}(C)} I(x, y)^{2} d x d y\right)
\end{aligned}
$$

where $\mu \geq 0$.

To compute the first variation of (1) it is useful to introduce an auxiliary function $\varphi: \Omega \rightarrow \Re$ such that $\varphi(\mathrm{x}, \mathrm{y})<0$ in $\Omega_{\mathrm{i}}$ and $\varphi(\mathrm{x}, \mathrm{y})>0$ in $\Omega_{\mathrm{e}}$ defining implicitly the curve $C$ as the zero level set of $\varphi$ [7]. Then the energy functional (1) can be rewritten using the Heaviside function $\mathrm{H}(\varphi)$, as

$$
\begin{aligned}
& F=\mu \int_{\Omega}|\nabla H(\varphi)| d x d y- \\
& {\left[\begin{array}{l}
A_{i} \log \left(\frac{1}{A_{i}} \int_{\Omega} I(x, y)^{2} H(\varphi) d x d y\right) \\
+A_{e} \log \left(\frac{1}{A_{e}} \int_{\Omega} I(x, y)^{2}(1-H(\varphi)) d x d y\right)
\end{array}\right]}
\end{aligned}
$$

where $A_{i}=\int_{\Omega} H(\varphi) d x d y \quad$ e $\quad A_{e}=\int_{\Omega}(1-H(\varphi)) d x d y \quad$ and length $\{\varphi=0\}=\int_{\Omega}|\nabla H(\varphi)| d x d y=\int_{\Omega} \delta(\varphi)|\nabla \varphi| d x d y$.

The associated Euler-Lagrange equations result:

$$
0=\delta(\varphi)\left[\begin{array}{l}
\mu \operatorname{div}\left(\frac{\nabla \varphi}{|\nabla \varphi|}\right)+ \\
+\log \left(\frac{1}{A_{i}} \int_{\Omega_{i}} I^{2} d x d y\right)+\frac{A_{i} I^{2}-\int_{\Omega_{i}} I^{2} d x d y}{\int_{\Omega_{i}}^{2} d x d y}- \\
-\log \left(\frac{1}{A_{e}} \int_{\Omega_{e}} I^{2} d x d y\right)-\frac{A_{e} I^{2}-\int_{\Omega_{e}} I^{2} d x d y}{\int_{\Omega_{e}} I^{2} d x d y}
\end{array}\right]
$$

The first term of the right hand side denotes the Euclidean curvature of level curves. It represents the speed of curve evolution in the normal direction.

In level set methods, the Euler-Lagrange equation for $\varphi$ acts only locally, on a few level curves around $\varphi=0$. To extend the evolution to all level sets of $\varphi$ we replace $\delta(\varphi)$ with $|\nabla \varphi|$. Thus the equation (3) will act on all the level curves, of course stronger on the zero level curve. From (3), we simply get the associated flow as:

$$
\partial_{t} \varphi=|\nabla \varphi|\left[\begin{array}{c}
\mu \operatorname{div}\left(\frac{\nabla \varphi}{\mid \nabla \varphi}\right)+ \\
+\log \left(\frac{1}{A_{i}} \int_{\Omega_{i}} I^{2} d x d y\right)+\frac{A_{i} I^{2}-\int_{\Omega_{i}} I^{2} d x d y}{\int_{\Omega_{i}} I^{2} d x d y}- \\
-\log \left(\frac{1}{A_{e}} \int_{\Omega_{e}} I^{2} d x d y\right)-\frac{A_{e} I^{2}-\int_{\Omega_{e}} I^{2} d x d y}{\int_{\Omega_{e}} I^{2} d x d y} \\
\frac{\left.\Omega^{2} x, y, 0\right)=\varphi_{0}(x, y)}{\frac{\delta(\varphi) \partial \varphi}{\mid \nabla \varphi \partial n}}
\end{array}\right]
$$

where $\varphi(\mathrm{x}, \mathrm{y}, 0)$ is an initial function with the property that its zero level set corresponds to the position of the initial front. Typically it is defined by $\varphi(x, y, 0)= \pm d$ where $d$ is the signed distance function from each point to the initial front [7]. $\frac{\delta(\varphi) \partial \varphi}{|\nabla \varphi| \partial n}=0$ are the boundary conditions. The 
evolution process will stop when the region probability terms of the inside regions do equal the terms of outside regions, up to regularization of boundaries.

In this study we applied this method to fifteen 2D echocardiographic images for the evaluation of chamber's size and extraction of chamber's area. Before testing the method on echocardiographic data we verified the assumption of Rayleigh distribution on all the images we used in this study. To this aim we compared the theoretical Rayleigh distribution with the histogram of the experimental ultrasound images as suggested in $[8,9]$. The estimation of the probability density function from the samples was performed applying the maximum likelihood algorithm and the root mean square error of the estimation was calculated for each fitting. To determine the reliability of the automated measurements versus the "gold standard", represented by manual tracings. Linear regression and Bland-Altman analyses were performed between the manually and automatically traced areas. Moreover, the manual contours were also overimposed and compared point-to-point to the corresponding automatic contours by the computation of the Hausdorff distance $[10,11]$. The Hausdorff distance measures the degree of mismatch between two sets of points, $\mathrm{C}_{1}$ and $\mathrm{C}_{2}$, by evaluating the distance between the point a of $\mathrm{C}_{1}$ that is farthest from any point $\mathrm{b}$ of $\mathrm{C}_{2}$ and vice versa: $H\left(C_{1}, C_{2}\right)=\max \left(h\left(C_{1}, C_{2}\right), h\left(C_{2}, C_{1}\right)\right)$ with $h\left(C_{1}, C_{2}\right)=\max _{a \in C_{1}} \min _{b \in C_{2}}\|a-b\|$.

\section{Results}

The simulations were performed in Matlab 6.1, on a Pentium IV personal computer, $3.06 \mathrm{Ghz}, 480 \mathrm{Mb}$ RAM. The time needed for the analysis of one image varied from few seconds to few minutes depending on the data size. The required operator interaction consists of one point selection in the image and then the analysis is completely automatic. In Figure 1 we present an example of the Rayleigh probability density function and the measured speckle pattern histogram of our image. For this example the root mean square error (rmse) of the estimation resulted in 0.019 . For the entire data set we processed, the mean rmse was 0.082 .

In our numerical experiments, we have used, for simplicity, $\Delta \mathrm{x}=\Delta \mathrm{y}=1$ and $\Delta \mathrm{t}=0.1$. Only the length parameter $\mu$, which has a scaling role, is not the same in all experiments. If we want to detect many objects and of any size, $\mu$ should be small. If we desire to detect only larger objects, then the parameter has to be bigger.

In Figure 2 we show the left ventricular chamber detection from a typical echocardiographic four chamber view acquisition. This computation is done with $\mu=1$.
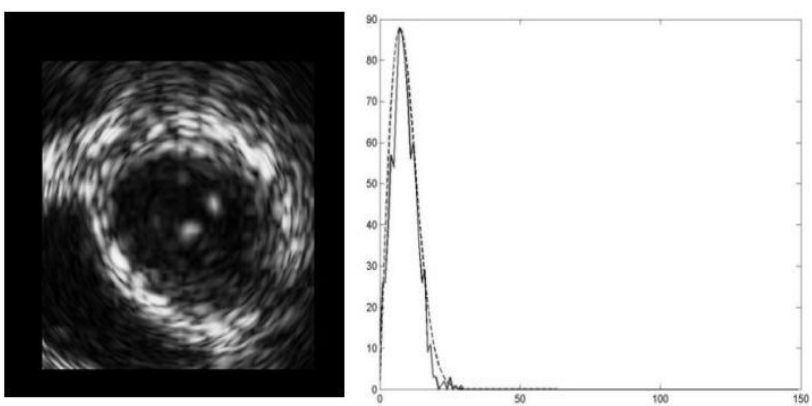

Figure 1. On the right panel, example of the fitting between the Rayleigh probability density function and the measured histogram of a uniform region of the B-scan image shown on the left.
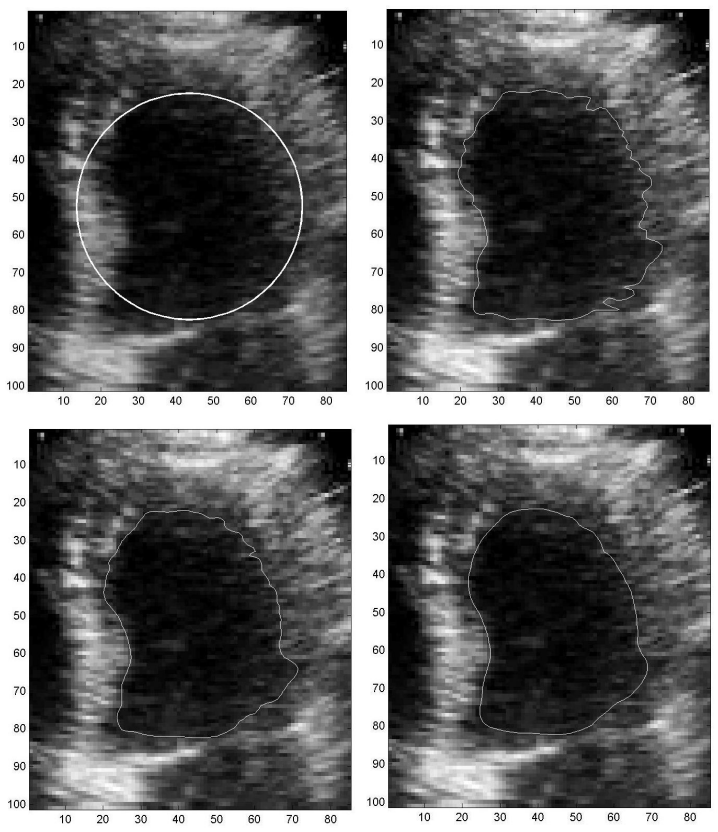

Figure 2. Initial condition (upper left) and some evolution steps of the segmentation of left ventricle from a typical echocardiographic four chamber view acquisition.

The comparison between the automated versus the manually traced contours resulted in a very good agreement in the areas calculated with both techniques, with an excellent correlation ( $\mathrm{r}=0.99$ ), and an absolute mean error, expressed in percentage of the "gold standard" value, of $2.7 \% \pm 2.4 \%$. Bland-Altman analysis resulted in a small negative bias of -31 pixels for the automated versus the manual measures, with the $95 \%$ confidence equal to 140 pixels. In Figure 3 linear regression (upper panel), BlandAltman (bottom panel) results are graphically reported. An example of the automated versus the manually traced contours is shown in Figure 4 where the good correspondence is visible. 

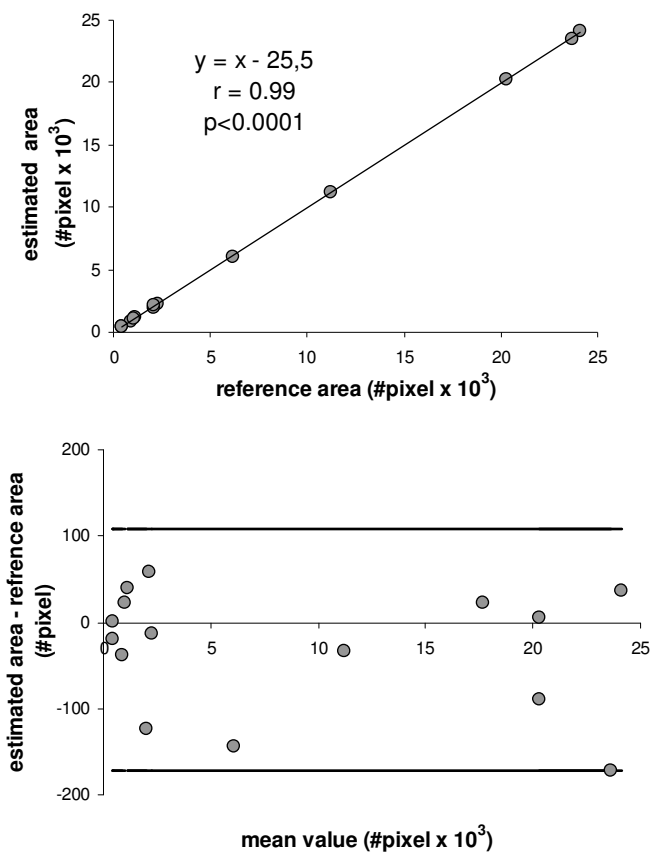

Figure 3. Linear regression analysis (left panel) and BlandAltman analysis (center panel) comparing area values obtained by manual tracings and by applying our maximum likelihood segmentation method.

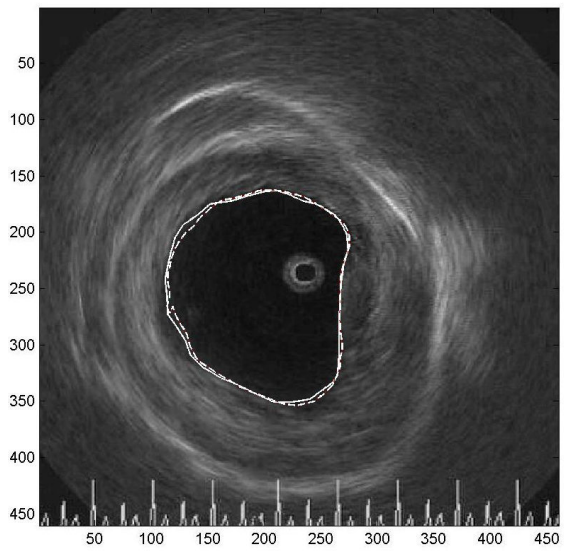

Figure 4. Example of the extracted contour obtained by the maximum likelihood segmentation method (white dashed line) compared to the manual tracing contour (white solid line) both applied to a cross sectional intra-cardiac acquisition with a catheter inserted through a sheath.

This was confirmed by the global results relevant to the Hausdorff distance where a mean value of 1.14 pixel (range: $0.36 \div 1.93$ pixels) and a mean maximum value of 4.33 pixels (range: $2 \div 8$ pixels) were found versus the gold standard.

\section{Discussion and conclusions}

A mathematical model and computational algorithm to segment ultrasound images has been presented. The technique is based on level set methods and exploits the a priori knowledge about the statistical distribution of image gray levels. Since ultrasound images show a low signal/noise ratio, it is often dangerous to compute differential quantities of the image signal. The method we propose is robust because it uses only integral quantities of the image and it does not introduce high frequency noise.

Only one parameter has to be set and it allows to choose easily the maximum curvature admissible in the segmentation. Moreover our method is considerably less exacting than manual segmentation.

\section{References}

[1] Kass M, Witkin A, Terzopoulos D. Snakes: Active Contour Models. International Journal of Computer Vision 1988; 1:321-331.

[2] Osher S, Sethian JA. Front Propagating with Curvature Dependent Speed: Algorithms Based on Hamilton Jacobi Formulation. J Comp Phys 1988;79:12-49.

[3] Chan TF, Vese LA. Active contours without edges. IEEE Trans Image Proc 2001;10(2):266-277.

[4] Bovik AC. On detecting edges in speckle imagery. IEEE Trans Acous Speech and Signal Proc 1988;36(10):16181627.

[5] Azzalini A. Statistical Inference-Based on the Likelihood, New York: Chapman and Hall, 1996.

[6] Chesnaud C, Refregier P, Boulet V. Statistical Region Snake-Based Segmentation Adapted to Different Physical Noise Models, IEEE Trans PAMI 1999;21(11):11451157.

[7] Sethian JA. Level Set Methods: Evolving Interface in Geometry, Fluid Mechanics, Computer Vision, and Materials Science, Cambridge University Press, 1996.

[8] Wagner RF, Smith SW, Sandrik JM, Lopez H. Statistics of speckle in ultrasound B-scans, IEEE Trans Sonics and Ultrasonics 1983;30(3):156-163.

[9] Burckhardt CB. Speckle in ultrasound B-mode scans, IEEE Trans Sonics and Ultrasonics 1978;25(1):1-6.

[10] Huttenlocher DP, Klanderman GA, Rucklidge WJ. Comparing images using the Hausdorff distance, IEEE Trans PAMI 1993;15(9):850-863.

[11] Belogay E, Cabrelli C, Molter U, Shonkwiler R. Calculating the Hausdorff distance between curves, Information Processing Letters 1997;64:17-22.

Address for correspondence

Alessandro Sarti

DEIS, University of Bologna

Viale Risorgimento 2, I-40136 Bologna

asarti@deis.unibo.it 